\title{
Deflation-activated sensors mediate pulmonary reflexes during acute pneumothorax
}

\author{
Jerry Yu* \\ Department of Medicine, University of Louisville, Robley Rex VAMC, Louisville, USA
}

\begin{abstract}
Patients with acute severe pneumothorax experience intense dyspnea, tachypnea, and hyperpnea, which directly correlate with severity of lung collapse. It is believed that during pneumothorax the collapse of the lung activates a group of pulmonary mechanical sensors, causing the reflex effects. Conventionally, pulmonary rapidly adapting receptors (RARs) were believed to be the responsible sensors. This review article provides evidence and arguments to support that pulmonary deflation activated receptors (DARs) are the responsible sensors, rather than RARs.
\end{abstract}

\section{Introduction}

One hundred and fifty years ago, Hering and Breuer observed that inflation of the lung suppresses inspiration and promotes expiration (Hering-Breuer inflation reflex), whereas deflation of the lung stimulates inspiration by increasing respiratory rate and inspiratory force (Hering-Breuer deflation reflex, HBDR) [1]. These two reflexes are mediated by the vagus nerve because they can be abolished by vagotomy. Some 20 years later, Head predicated that deflation stimulates some pulmonary sensors to cause the HBDR, because lung deflation produces stronger stimulation on breathing than vagotomy, although both procedures remove inhibitory signals (associated with lung inflation) in the vagus nerve [1]. With development of electrical recording of a single fiber of the vagal afferent nerve, the behavior of airway sensors could be studied. Two types of airway mechanosensors, pulmonary RARs and SARs (slowly adapting receptors), were identified [2]. As their names indicate, the former adapts rapidly, whereas the latter adapts slowly during lung inflation with constant pressure or volume. Yet, many RARs and SARs are also stimuIated during lung deflation (Figure 1). Soon after the identification of sensors, SARs were established as the sensors responsible for the Hering-Breuer inflation reflex [3]. However, the exact type of sensors responsible for the HBDR has never been established. By the turn of the century, all scientists agreed that decreases in SAR activities during the lung deflation phase contribute to the HBDR. This passive contribution is due to removal of the inhibitory effect. In addition, most scientists also believed that activation of RARs was responsible for the HBDR [4-6], and thus responsible for the pneumothorax induced reflex effects. Recent research data support the existence of another group of mechanosensors, the DARs, which are proposed as responsible for the HBDR [7].

\section{Conventional belief (RARs as the candidate for HBDR)}

Due to a lack of detailed information about structure-function relationships within the pulmonary sensory system, researchers believe that one single afferent fiber of the vagus nerve is connected with only one mechanosensor (the single-sensor theory) [1]. In this scenario, mechanosensors of the same type send their afferent signals to specific parts of the central nervous system to evoke reflex responses. That

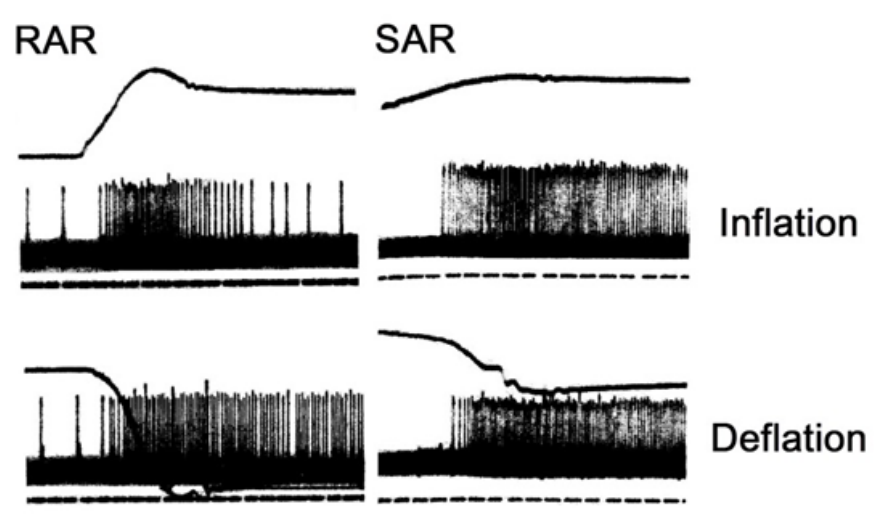

Figure 1. Responses of RAR (left) and SAR (right) units to lung inflation (up panels) and deflation (low panels). During inflation, initially the RAR discharges vigorously, which adapts quickly, while the SAR adapts slowly with a continuous high discharge frequency. During deflation, however, both units behave similarly, discharging vigorously with slow adaptation. The potential for these same responses from the RAR and SAR during deflation to contribute to the Hering-Breuer deflation reflex (and thus the pneumothorax induced reflexes) is the focus of this article. Traces: top, lung volume; middle, action potentials; bottom (dotted lines), time marks. (Adapted from figure 2 of [1], which is originated from Figures 2 and 8 of [2])

is, activation of a given type of sensors elicits a unique response. In this scenario, there were many sub-groups of RARs and SARs. Some respond only to inflation and the others respond to both inflation and deflation. Clearly, in the single-sensor theory, pure DARs (sensors that respond only to deflation) would be the best candidates for producing the HBDR [1]. Therefore, researchers were actively searching for DARs [8]. However, pure DARs are few in large animals (such as dogs, cats and rabbits) and thus it is unlikely that they can produce significant reflex effects [9]. Although a significant number of DARs is present in

*Correspondence to: Yu J, Robley Rex VA Medical Center, Louisville, KY, 40206 and Departments of Medicine, University of Louisville, Louisville, KY 40292, USA, Tel: 502-852-5146; Fax: 502-852-1359; E-mail: j0yu0001@louisville.edu

Received: October 19, 2018; Accepted: October 29, 2018; Published: October 31,2018 
rodents, this cannot explain reflex effects in large animals. Since SARs have lower activation thresholds than RARs during lung inflation, a continuously low level of lung inflation (which activates SARs but not RARs) produces long lasting inhibitory effects on breathing. The time course involved is far beyond a significant portion of RAR activities has adapted. On the other hand, the high level of lung inflation required to activate RARs produces transient excitatory effects on breathing, which fits the time course of activation of RARs [10]. This supports the theory that activation of SARs inhibits, whereas activation of RARs stimulates inspiration. Since a higher percentage of RARs (2/3) than SARs $(1 / 3)$ responds to deflation, and lung deflation stimulates inspiration, RARs were accepted as the sensors responsible for the HBDR [2].

\section{Challenges to the conventional beliefs}

Clearly, RARs are the best candidates in the single-sensor theory. However, stating that RARs cause the HBDR is problematic. One third of RARs do not respond to lung deflation and many RARs adapt rapidly during deflation. These RARs cannot be responsible for the HBDR. Since the HBDR is long lasting, similar to the rationale that only SARs can be responsible for Hering-Breuer inflation reflex, only those sensors that adapt slowly during lung deflation can be responsible for the HBDR. Furthermore, RARs are identified by their response to lung inflation. Using an inflation stimulus to establish a deflation reflex is not justified. Yet, many SARs respond to lung deflation with a slow adaptation (Figure 1). Their role in the HBDR has never been discussed [1], presumably because these activities were considered to be inhibitory [11]. A discussion of their reflex effects would certainly cause a dilemma. The number of SARs is four to ten times of that of RARs [5]. During deflation, total inputs from SARs should be more than those from RARs. If deflation-induced effects from RARs and SARs are the same in strength but opposite in direction, then the net reflex effects will be inhibitory. These unsolved problems can be easily tackled if a multi-sensor theory is accepted (please see below).

\section{New interpretation (sensory unit concept)}

With recent advances in the field of pulmonary vagal sensory receptors, the single-sensor theory is nolonger tenable. Morphologically, a parent axon has been found to split into many branches and supply to several sensors [12]. Thus, electrical activities recorded from a single fiber may come from multiple sensors. Therefore, a single fiber recording is a single unit recording instead of a single sensor recording. Indeed, during single fiber recording we found multiple receptive fields. By blocking one of the fields with lidocaine, we found that each field operates not as a contributor but as an encoder [13], because in many cases the blockade did not change the peak discharge frequency. If each field was a contributor, after blockade the peak frequency would inevitably decrease. Furthermore, the inflation and deflation responses could be selectively blocked by injection of lidocaine into a receptive field [7], supporting the theory that inflation and deflation responses were coming from different types of sensors (inflation activated sensors vs deflation activated sensors). Moreover, neural activities from a single fiber recording may behave in a combined pattern of RARs and SARs (Figure 5 in reference 14; and Figure 6 in reference 7), which can also be selectively blocked by injection of lidocaine into a receptive field [7].

Based on the above advances, a multi-sensor theory has been proposed [1]. In this scenario, a single afferent fiber of the vagus nerve is connected with a sensory unit instead of an individual sensor. Like its counterpart of the motor unit, which sends signals simultaneously to different effectors to cause a unified motor action, a sensory unit receives and integrates information from many sensors. A sensory unit contains multiple sensors. These sensors can be the same or different types (SARs, RARs, and DARs). Each type senses a particular force. Thus, a sensory unit's behavior is dictated by the composition of its sensors. If a sensory unit contains many homogeneous SARs (or DARs) only, then it will respond to inflation (or deflation) only. If the unit contains both SARs and DARs, it will respond to both inflation and deflation as shown in the right column of Figure 1 (if the DARs are the slowly adapting type). If a unit contains both RAR and SAR types, it will behave in a combined pattern of RARs and SARs. These results justify the classification of DARs as a separate group of sensors including pure DARs and DARs that share axons with SARs and/or RARs. DARs can be further subdivided into rapidly adapting or slowly adapting ones [1]. The rapidly and slowly adapting DARs may co-exist in a sensory unit to produce a combined response to deflation (please see $B, F$, and $J$ of figure 3 in reference 7 ). Only when a unit contains slowly adapting DARs, can it actively and substantially contribute to the HBDR.

\section{Summary}

Acute pneumothorax causes an intense dyspneic sensation. Patients struggle for air and present with rapid deep breathing, or rapid shallow breathing if chest movement causes pain. These reflex effects are initiated from pulmonary mechanosensors. With the singlesensor theory, RARs were thought to be responsible for these reflex effects. With identification of DARs and recognition of multi-sensors in a sensory unit, slowly adapting DARs (which exist either purely or connected with RARs and/or SARs) are believed to be the airway sensors that are responsible for the pneumothorax induced reflex effects.

\section{Acknowledgement}

A merit review award (PULM-024-175) from the US Department of Veteran Affairs.

\section{References}

1. Yu J (2016) Deflation-activated receptors, not classical inflation-activated receptors, mediate the Hering-Breuer deflation reflex. J Appl Physiol 121: 1041-1046. [Crossref]

2. Knowlton GC, Larrabee MG (1946) A unitary analysis of pulmonary volume receptors Am J Physiol 147: 100-114. [Crossref]

3. Widdicombe JG (1964) Respiratory reflexes. Handbook of Physiology, Respiration. Washington, D.C. Am Physiol Soc 1964: 585-630.

4. Coleridge HM, Coleridge JCG (1986) Reflexes evoked from tracheobronchial tree and lungs. In: Cherniack NS, Widdicombe JG, editors. Handbook of Physiology. Bethsda: American Physiological Society 1: 395-430.

5. Sant'Ambrogio G (1982) Information arising from the tracheobronchial tree of mammals. Physiol Rev 62: 531-569. [Crossref]

6. Widdicombe JG (2001) Airway receptors. Respir Physiol 125: 3-15

7. Liu J, Yu J (2013) Spectrum of myelinated pulmonary afferents (II). Am J Physiol Regul Integr Comp Physiol 305: R1059-R1064. [Crossref]

8. Paintal AS (1973) Vagal sensory receptors and their reflex effects. Physiol Rev 53 159-227. [Crossref]

9. Widdicombe JG (1954) Receptors in the trachea and bronchi of the cat. J Physiol 123 71-104.

10. Larrabee MG, Knowlton GC (1946) Excitation and inhibition of phrenic motoneurones by inflation of the lungs. Am J Physiol 147: 90-99.

11. Bartlett DJ, Jeffery P, Sant'Ambrogio G, Wise JC (1976) Location of stretch receptors in the trachea and bronchi of the dog. J Physiol 258: 409-420.

12. Liu J, Song N, Guardiola J, Roman J, Yu J (2016) Slowly adapting sensory units have more receptors in large airways than in small airways in rabbits. Front Physiol 7: 588 [Crossref] 
Yu J (2018) Deflation-activated sensors mediate pulmonary reflexes during acute pneumothorax

13. Yu J, Zhang J (2004) A single pulmonary mechano-sensory unit possesses multiple encoders in rabbits. Neurosci Lett 362: 171-175. [Crossref]
14. Yu J (2000) Spectrum of myelinated pulmonary afferents. Am J Physiol Regul Integr Comp Physiol 279: R2142-R2148. [Crossref]

Copyright: $\mathbb{0} 2018$ Yu J. This is an open-access article distributed under the terms of the Creative Commons Attribution License, which permits unrestricted use, distribution, and reproduction in any medium, provided the original author and source are credited. 